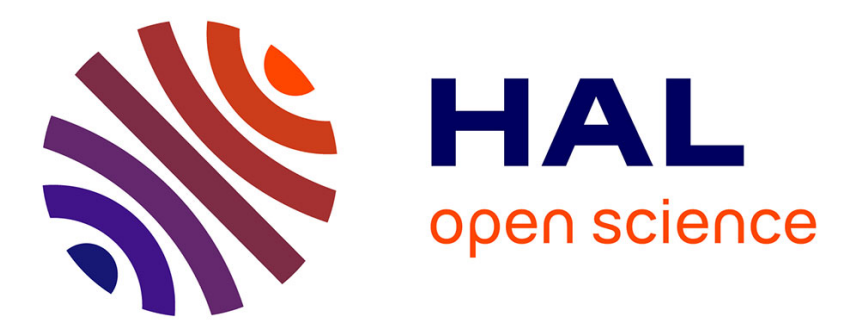

\title{
Expérimentation d'une approche parallèle en calcul de structures
}

Jean-Yves Cognard, David Dureisseix, Pierre Ladevèze, Philippe Lorong

\section{To cite this version:}

Jean-Yves Cognard, David Dureisseix, Pierre Ladevèze, Philippe Lorong. Expérimentation d'une approche parallèle en calcul de structures. Revue Européenne des Éléments Finis, 1996, 5 (2), pp.197220. 10.1080/12506559.1996.10511217 . hal-00321329

\section{HAL Id: hal-00321329 \\ https://hal.science/hal-00321329}

Submitted on 9 Dec 2017

HAL is a multi-disciplinary open access archive for the deposit and dissemination of scientific research documents, whether they are published or not. The documents may come from teaching and research institutions in France or abroad, or from public or private research centers.
L'archive ouverte pluridisciplinaire HAL, est destinée au dépôt et à la diffusion de documents scientifiques de niveau recherche, publiés ou non, émanant des établissements d'enseignement et de recherche français ou étrangers, des laboratoires publics ou privés. 


\title{
Expérimentation d'une approche parallèle en calcul des structures
}

\author{
J.-Y. Cognard ${ }^{1}$, D. Dureisseix ${ }^{1}, \mathrm{P}_{\text {. Ladevèze }}{ }^{1}$, and $\mathrm{Ph}$. \\ Lorong $^{1}$ \\ ${ }^{1}$ Laboratoire de Mécanique et Technologie, (E.N.S. de \\ Cachan / Université Paris 6 / C.N.R.S.), 61 Avenue du \\ Président Wilson, 94235 CACHAN CEDEX
}

\begin{abstract}
Nous décrivons les grandes lignes d'une formulation mécanique qui propose une approche "parallèle" pour l'analyse des structures en petites perturbations et nous nous restreignons ici au cas de l'élasticité linéaire. La représentation du milieu que nous introduisons est celle d'un assemblage de sous-structures et d'interfaces. Les liaisons et contacts divers entre les sous-structures sont pris en compte par les interfaces qui possèdent leur comportement propre. La convergence est démontrée sous des conditions classiques de stabilité. Quelques exemples illustrent le comportement de l'approche proposée sur ordinateurs à architecture parallèle.

We describe the headlines of a mechanical formulation that inforce a "parallel" approach for structural analysis in small perturbations, restricted here to linear elasticity. The description of the medium is an assembly of substructures and interfaces. Liaisons and contacts between substructures are treated by the interfaces which possess their own behaviour. Convergence is proved under classical stability assumptions. Several exemples figure out the behaviour of the propounded approach on parallel architecture computers.

This is a preprint of the article published by Taylor \& Francis in Revue Européenne des Éléments Finis / European Journal of Computational Mechanics 5(2) :197-220, 1996, available online : http://www.tandfonline. com/10.1080/12506559.1996.10511217.
\end{abstract}

Keywords : structures, calcul, parallélisme computation, parallelism 


\section{Introduction}

Les ordinateurs qui proposent aujourd'hui les plus grandes puissances de traitement sont presque tous parallèles. L'utilisation de ces derniers pour l'analyse, notamment non linéaire, des structures, constitue donc un atout considérable. L'écriture d'algorithmes adaptés au parallélisme, ou la transcription d'algorithmes séquentiels existants, n'est en général pas immédiate, et de nombreuses recherches sont menées dans ce domaine, DÉB 90], FAR 91, PAN 93, BUO 93, NOO 94.

Ce travail traite d'une approche "parallèle", fortement mécanique, des problèmes d'élastoplasticité, de viscoplasticité, ...., en petites perturbations, proposée dans LAD 96] ; des présentations plus succinctes pourront être trouvées dans [LAD 93] et LAD 92b. Cette approche peut être rangée dans la famille des méthodes de décomposition de domaine dont traitent [ESC 94, [FAR 94a] et YAG 93] par exemple.

La vocation de cet article est de présenter une première mise en œuvre numérique et informatique, en se restreignant essentiellement à des problèmes linéaires. Les exemples traités permettent d'appréhender les possibilités de cette approche, qui pour les problèmes linéaires et les choix de paramètres que nous avons pris se confond avec les approches proposées dans LAD 85, [LIO 90] et GLO 90.

\section{Problème de référence}

Dans les trois prochaines parties, les auteurs ont choisi de présenter à la fois le problème de référence et l'algorithme de résolution indépendemment du choix de la discrétisation; ainsi le modèle adopté est celui du milieu continu.

On se place dans le cadre des petites perturbations et dans la situation particulière où l'on s'intéresse uniquement à la configuration finale occupée par la structure. Aussi, le temps n'est pas un paramètre du problème.

La structure élastique étudiée occupe un domaine $\Omega$ dont la frontière est notée $\partial \Omega$. Elle est soumise à un chargement composé de force de volume $f_{d}$, et de forces surfaciques $\underline{F}_{d}$ sur la portion de frontière $\partial_{2} \Omega$ de $\partial \Omega$. Sur la portion de frontière $\partial_{1} \Omega$, complémentaire de $\partial_{2} \Omega$, le déplacement $\underline{U}_{d}$ est imposé (figure 1).

Le problème à résoudre consiste alors à trouver un couple $(\underline{U}(\underline{M}) ; \boldsymbol{\sigma}(\underline{M}))$, $M \in \Omega$, pour lequel :

- Le champ de déplacement $\underline{U}$ est Cinématiquement Admissible, c'est à dire :

$$
\underline{U}_{\mid \partial_{1} \Omega}=\underline{U}_{d}+\text { "régularité" }
$$

L'ensemble des champs de déplacement Cinématiquement Admissibles sera noté $\mathcal{U}_{a d}$. La "régularité" consiste ici essentiellement à avoir des champs à énergie finie. 


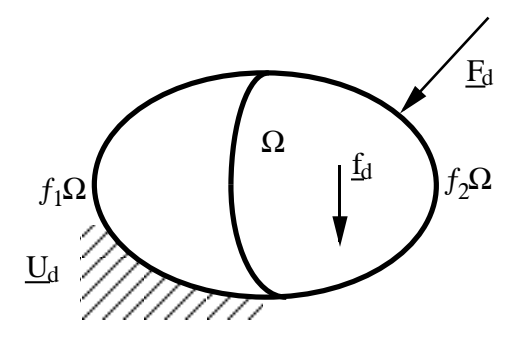

FiguRE 1: Problème de référence

- Le champ de contrainte $\boldsymbol{\sigma}$ est Statiquement Admissible, c'est à dire :

$$
\forall \underline{U}^{\star} \in \mathcal{U}^{0},-\int_{\Omega} \operatorname{Tr}\left[\boldsymbol{\sigma} \varepsilon\left(\underline{U}^{\star}\right)\right] d \Omega+\int_{\Omega} \underline{f}_{d} \cdot \underline{U}^{\star} d \Omega+\int_{\partial_{2} \Omega} \underline{F}_{d} \cdot \underline{U}^{\star} d S=0
$$

où $\mathcal{U}^{0}$ est l'ensemble des champs $\underline{U}^{\star}$ Cinématiquement Admissibles pour des conditions homogènes. L'ensemble des champs de contrainte Statiquement Admissibles sera quant à lui noté $\mathcal{S}_{a d}$.

- La relation de comportement est vérifiée :

$$
\sigma=\mathbf{K} \varepsilon(\underline{U})
$$

où $\mathbf{K}$ est l'opérateur de Hooke caractéristique du matériau.

\section{Formulation du problème intégrant une décomposition en sous-structures}

La recherche d'un champ de contrainte Statiquement Admissible sur toute la structure $\Omega$, est un problème global en variable d'espace. Afin de rompre cette globalité, et ainsi pouvoir distribuer les tâches en parallèle, on introduit une partition du milieu en deux entités : les sous-structures et les interfaces. Les interfaces assurent les liaisons entre les sous-structures. Elles ont un comportement qui traduit le type d'interaction présent entre sousstructures adjacentes et peuvent être considérées comme des structures à part entière, même si elles sont surfaciques.

Lorsque la structure étudiée résulte d'un assemblage de sous-structures, la partition s'introduit naturellement en utilisant les surfaces de jonction entre les diverses sous-structures. Lorsqu'elle est composée d'une ou d'un nombre réduit de sous-structures massives, la partition peut se faire de façon artificielle au sein des sous-structures, par exemple en utilisant une décomposition automatique [FAR 88, [PAD 91]. Cette partition peut alors répondre à des impératifs d'optimisation (vitesse de convergence de l'algorithme) ainsi qu'à l'équilibrage des charges entre processeurs parmi lesquels seront distribuées les sous-structures. 


\subsection{Comportement des sous-structures}

Considérons une sous-structure $\Omega^{E}$, dont la frontière est noté $\partial \Omega^{E}$. Elle doit être en équilibre avec un environnement qui est composé de forces de volume $\underline{f}_{d}^{E}$ (appliquées sur $\Omega^{E}$ ) ainsi que de déplacements $\underline{W}^{E}$ et de densités d'efforts $\underline{F}^{E}$ exercés par les interfaces qui entourent $\Omega^{E}$ (appliqués sur $\partial \Omega^{E}$ ) (figure 2 .
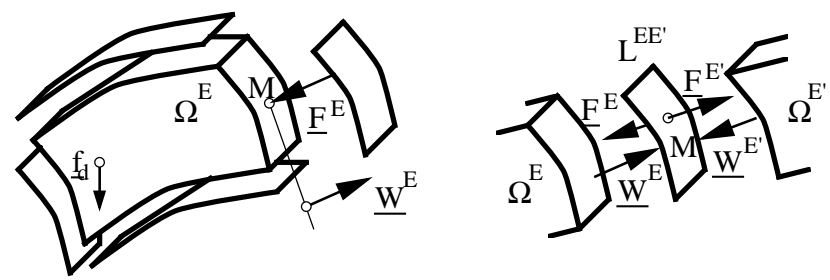

Figure 2: Sous-structure et interface

Vis à vis de la sous-structure $\Omega^{E},\left(\underline{U}^{E}, \underline{W}^{E} ; \boldsymbol{\sigma}^{E}, \underline{F}^{E}\right)$ doit vérifier :

- Les équations de liaison : le couple $\left(\underline{U}^{E}, \underline{W}^{E}\right)$ est Cinématiquement Admissible au sens de la sous-structure $\Omega^{E}$

$$
\begin{array}{r}
\underline{U}^{E} \in \mathcal{U}_{a d}^{E} \\
\underline{U}^{E}{ }_{\mid \partial \Omega^{E}}=\underline{W}^{E}
\end{array}
$$

où $\mathcal{U}_{a d}^{E}$ est l'ensemble des champs de déplacement définis sur $\Omega^{E}$ "réguliers".

- Les équations d'équilibre : le couple $\left(\boldsymbol{\sigma}^{E}, \underline{F}^{E}\right)$ doit être Statiquement Admissible au sens de la sous-structure $\Omega^{E}$, c'est à dire :

$$
\begin{aligned}
& \forall \underline{U}^{\star} \in \mathcal{U}_{a d}^{E}, \\
& -\int_{\Omega^{E}} \operatorname{Tr}\left[\boldsymbol{\sigma}^{E} \varepsilon\left(\underline{U}^{\star}\right)\right] d \Omega+\int_{\Omega^{E}} \underline{f}_{d}^{E} \cdot \underline{U}^{\star} d \Omega+\int_{\partial \Omega^{E}} \underline{F}^{E} \cdot \underline{U}^{\star} d S=0,
\end{aligned}
$$

- La relation de comportement :

$$
\boldsymbol{\sigma}^{E}=\mathbf{K} \varepsilon\left(\underline{U}^{E}\right)
$$

\subsection{Comportement des interfaces}

De l'environnement des sous-structures, on déduit celui des interfaces : il est composé d'efforts $\underline{F}$ et de déplacements $\underline{W}$.

Considérons la liaison $L^{E E^{\prime}}$ qui relie les sous-structures $\Omega^{E}$ et $\Omega^{E^{\prime}}$ (la figure 2 précise les notations). Son état est caractérisé par les valeurs sur sa surface $\Gamma^{E E^{\prime}}$ de $\left(W^{E} ; F^{E}\right)$ et $\left(W^{E^{\prime}} ; F^{E^{\prime}}\right)$ qui sont respectivement les couples déplacement-effort décrivant son action sur les sous-structures $\Omega^{E}$ et $\Omega^{E^{\prime}}$. 
Le comportement d'une telle interface dépend du type de liaison entre sous-structures qu'elle représente. Par exemple :

- Liaison parfaite

$$
\underline{F}^{E}+\underline{F}^{E^{\prime}}=0 \quad \text { et } \quad \underline{W}^{E}=\underline{W}^{E^{\prime}} \quad \text { sur } \quad \Gamma^{E E^{\prime}}
$$

Ce type de liaison est, en particulier, utilisé lors d'une partition artificielle au sein de la structure étudiée.

- Liaison "efforts imposés" ou "déplacements imposés"

Elles peuvent être traitées comme des liaisons parfaites. Toutefois, les conditions en déplacement, ou en effort, sont imposées au "bâti" $B$, qui est une sous-structure particulière. En plus des équations relatives aux interfaces parfaites, on a les équations qui résument le comportement du bâti, soit :

- frontière à déplacement imposé : $\underline{U}^{B}=\underline{U}_{d}$,

- frontière à effort imposé : $\underline{F}^{B}=-\underline{F}_{d}$.

- Contact unilatéral sans frottement et sans jeu

Soient $\underline{n}^{E E^{\prime}}$ la normale unitaire à $\Gamma^{E E^{\prime}}$ au point courant, orientée de $\Omega^{E}$ vers $\Omega^{E^{\prime}}$ et $\boldsymbol{\pi}$ l'opérateur de projection orthogonale correspondant. On a sur $\Gamma^{E E^{\prime}}$.

$$
\begin{gathered}
\underline{F}^{E}+\underline{F}^{E^{\prime}}=0 \text { et } \pi \underline{F}^{E}=\pi \underline{F}^{E^{\prime}}=0 \\
\underline{n}^{E E^{\prime}} \cdot\left(\underline{W}^{E^{\prime}}-\underline{W}^{E}\right) \geq 0 \text { et } \underline{n}^{E E^{\prime}} \cdot \underline{F}^{E^{\prime}} \geq 0 \\
\left(\underline{n}^{E E^{\prime}} \cdot\left(\underline{W}^{E^{\prime}}-\underline{W}^{E}\right)\right)\left(\underline{n}^{E E^{\prime}} \cdot \underline{F}^{E^{\prime}}\right)=0
\end{gathered}
$$

- Écriture générale du comportement d'une interface

Ayant l'équilibre local $\underline{F}^{E}+\underline{F}^{E^{\prime}}=0$, considérons la discontinuité en déplacement $\underline{W}^{E E^{\prime}}=\underline{W}^{E^{\prime}}-\underline{W}^{E}$ sur l'interface $L^{E E^{\prime}}$. Il s'agit d'une grandeur cinématique analogue à une déformation. $\underline{F}^{E E^{\prime}}=-\underline{F}^{E}=$ $\underline{F}^{E^{\prime}}$ en est la grandeur conjuguée, ici une force. Ainsi la formulation fonctionnelle de la relation de comportement de l'interface est alors donnée par :

$$
\underline{F}^{E E^{\prime}}=\mathbb{H}\left(\underline{W}^{E E^{\prime}}\right)
$$

$\mathbb{H}$ est un opérateur caractéristique de l'interface.

L'idée de modéliser un contact, une liaison, par une entité mécanique surfacique est souvent utilisée dans le domaine du calcul. Pour les méthodes de décomposition de domaine, elle a été introduite par J. Ladevèze dans [LAD 85]. 


\section{Approche parallèle}

\subsection{Cadre mécanique}

L'assemblage étudié est constitué de sous-structures $\Omega^{E}, E \in \mathrm{E}$, et d'interfaces $L^{E E^{\prime}}$. L'état du milieu est caractérisé par $\mathbf{s}=\bigcup_{E \in E} \mathbf{s}^{E}$,

avec $\mathbf{s}^{E}=\left(\underline{U}^{E}, \underline{W}^{E} ; \boldsymbol{\sigma}^{E}, \underline{F}^{E}\right) . \underline{W}^{E}$ et $\underline{F}^{E}$ sont définis sur $\partial \Omega^{E}$ et les autres quantités dans $\Omega^{E}$. L'espace fonctionnel introduit est noté $\mathbf{S}$.

Le problème à résoudre consiste alors à trouver $\mathbf{S} \in \mathbf{S}$ tel que :

- toutes les équations associées aux sous-structures soient vérifiées,

- toutes les équations associées aux interfaces soient vérifiées.

Afin de résoudre ce problème (il s'agit ici d'une situation dégénérée où on étudie uniquement la configuration finale), nous utilisons une approche dite à grand incrément de temps LAD 92a dont une première spécificité est de partager les équations du problème en deux groupes :

- un groupe d'équations linéaires, éventuellement globales en variable d'espace par sous-structure, qui comprend

- pour chaque interface $L^{E E^{\prime}}$ : néant,

- pour chaque sous-structure $\Omega^{E}$ : $\quad$ relation de comportement (3), équations de liaison (1) et d'équilibre (2).

- un groupe d'équations locales en variable d'espace, éventuellement non linéaires, parmi lesquelles on choisit de faire figurer

- pour chaque sous-structure $\Omega^{E}$ : néant,

- pour chaque interface $L^{E E^{\prime}}$ : $\quad$ comportement de l'interface,

L'ensemble des solutions $\mathbf{s}$ (appartenant à $\mathbf{S}$ ) du premier groupe d'équation est noté $\mathbf{A}_{d}$. L'espace des solutions du second groupe est désigné par $\boldsymbol{\Gamma}$. Le problème initial peut alors être décrit comme la recherche de l'intersection $\mathbf{s}_{e x}$ des deux variétés $\mathbf{A}_{d}$ et $\boldsymbol{\Gamma}$.

\subsection{Approche à grand incrément de temps}

La méthode de résolution est itérative; elle utilise des directions de recherche qui sont les paramètres de la méthode. Alternativement, elle construit un point de $\mathbf{A}_{d}$ et un point de $\boldsymbol{\Gamma}$ jusqu'à la convergence pratique. Chaque itération comprend deux étapes, l'une est dite locale et l'autre linéaire (figure 3).

- Étape locale $: \mathbf{s}_{n} \rightarrow \hat{\mathbf{s}}_{n+1 / 2}$, avec

$$
\hat{\mathbf{s}}_{n+1 / 2} \in \boldsymbol{\Gamma}
$$

$\left(\hat{\mathbf{s}}_{n+1 / 2}-\mathbf{s}_{n}\right)$ appartenant de plus à la direction de recherche $\mathbf{E}^{+}$, ce que l'on écrit sous la forme :

$$
\left(\underline{\hat{F}}_{n+1 / 2}^{E}-\underline{F}_{n}^{E}\right)-\mathbf{k}^{+}\left(\underline{\hat{W}}_{n+1 / 2}^{E}-\underline{W}_{n}^{E}\right)=0
$$




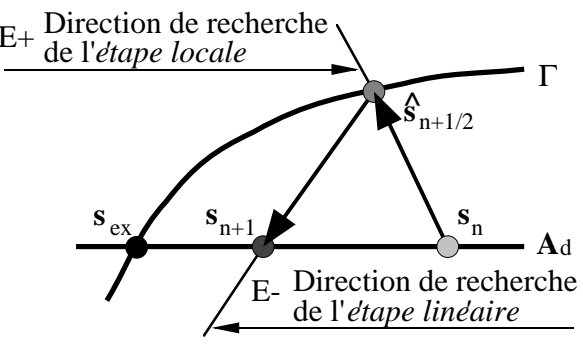

Figure 3: Principe de la méthode

$$
\left(\underline{\hat{E}}_{n+1 / 2}^{E^{\prime}}-\underline{F}_{n}^{E^{\prime}}\right)-\mathbf{k}^{+}\left(\underline{\hat{W}}_{n+1 / 2}^{E^{\prime}}-\underline{W}_{n}^{E^{\prime}}\right)=0
$$

en tout point de toute interface $L^{E E^{\prime}}$.

$\mathbf{k}^{+}$étant une opérateur local en variables d'espace, cette étape ne porte que sur des quantités locales; elle est donc parfaitement parallélisable. En particulier pour une interface parfaite, on a explicitement :

$$
\begin{gathered}
\underline{\hat{W}}_{n+1 / 2}^{E}=\underline{\hat{W}}_{n+1 / 2}^{E^{\prime}}=\frac{1}{2}\left[\left(\underline{W}_{n}^{E}+\underline{W}_{n}^{E^{\prime}}\right)-\left(\mathbf{k}^{+}\right)^{-1}\left(\underline{F}_{n}^{E}+\underline{F}_{n}^{E^{\prime}}\right)\right] \\
\underline{\hat{F}}_{n+1 / 2}^{E}=-\underline{\hat{F}}_{n+1 / 2}^{E^{\prime}}=\frac{1}{2}\left[\left(\underline{F}_{n}^{E}-\underline{F}_{n}^{E^{\prime}}\right)-\mathbf{k}^{+}\left(\underline{W}_{n}^{E}-\underline{W}_{n}^{E^{\prime}}\right)\right]
\end{gathered}
$$

Un choix possible pour la forme de $\mathbf{k}^{+}$est $\mathrm{k}^{+} \mathbb{I}_{d}$, où $\mathrm{k}^{+}$est un scalaire positif.

Pour une interface de contact sans frottement et sans jeu, la direction de recherche permet d'écrire pour la composante normale :

$$
\begin{gathered}
\underline{n}^{E E^{\prime}} \cdot\left(\underline{\hat{W}}_{n+1 / 2}^{E^{\prime}}+\underline{\hat{W}}_{n+1 / 2}^{E}\right)=\underline{n}^{E E^{\prime}} \cdot\left(\underline{W}_{n}^{E^{\prime}}+\underline{W}_{n}^{E}\right)-\frac{1}{\mathrm{k}^{+}} \underline{n}^{E E^{\prime}} \cdot\left(\underline{F}_{n}^{E^{\prime}}+\underline{F}_{n}^{E}\right)=b \\
-\underline{n}^{E E^{\prime}} \cdot\left(\underline{\hat{F}}_{n+1 / 2}^{E^{\prime}}-\underline{\hat{F}}_{n+1 / 2}^{E}\right)+\mathrm{k}^{+} \underline{n}^{E E^{\prime}} \cdot\left(\underline{\hat{W}}_{n+1 / 2}^{E^{\prime}}-\underline{\hat{W}}_{n+1 / 2}^{E}\right)= \\
=\underline{\mathrm{k}}^{+} \underline{n}^{E E^{\prime}} \cdot\left(\underline{W}_{n}^{E^{\prime}}-\underline{W}_{n}^{E}\right)-\underline{n}^{E E^{\prime}} \cdot\left(\underline{F}_{n}^{E^{\prime}}-\underline{F}_{n}^{E}\right)=a
\end{gathered}
$$

Le comportement de l'interface permet d'écrire :

$$
\begin{gathered}
-\underline{n}^{E E^{\prime}} \cdot\left(\underline{\hat{F}}_{n+1 / 2}^{E^{\prime}}-\underline{\hat{F}}_{n+1 / 2}^{E}\right)=-2 \underline{n}^{E E^{\prime}} \cdot \underline{\hat{F}}_{n+1 / 2}^{E^{\prime}} \leq 0 \\
\underline{n}^{E E^{\prime}} \cdot\left(\underline{\hat{W}}_{n+1 / 2}^{E^{\prime}}-\underline{\hat{W}}_{n+1 / 2}^{E}\right) \geq 0
\end{gathered}
$$

et d'en déduire que :

$$
\begin{gathered}
\underline{n}^{E E^{\prime}} \cdot \underline{\hat{F}}_{n+1 / 2}^{E^{\prime}}=-\underline{n}^{E E^{\prime}} \cdot \underline{\hat{F}}_{n+1 / 2}^{E}=\frac{1}{2}|-a| \\
\underline{n}^{E E^{\prime}} \cdot \underline{\hat{W}}_{n+1 / 2}^{E^{\prime}}=\frac{1}{2}\left(b+\frac{1}{\mathrm{k}^{+}}|a|\right)
\end{gathered}
$$




$$
\underline{n}^{E E^{\prime}} \cdot \underline{\hat{W}}_{n+1 / 2}^{E}=\frac{1}{2}\left(b-\frac{1}{\mathrm{k}^{+}}|a|\right)
$$

Pour les composantes tangentielles,

$$
\pi \underline{\hat{F}}_{n+1 / 2}^{E^{\prime}}=\pi \underline{\hat{F}}_{n+1 / 2}^{E}=0
$$

et la direction de recherche donne :

$$
\begin{gathered}
\boldsymbol{\pi} \underline{\hat{W}}_{n+1 / 2}^{E^{\prime}}=\boldsymbol{\pi} \underline{W}_{n}^{E^{\prime}}-\frac{1}{\mathrm{k}^{+}} \boldsymbol{\pi} \underline{F}_{n}^{E^{\prime}} \\
\boldsymbol{\pi} \underline{\hat{W}}_{n+1 / 2}^{E}=\boldsymbol{\pi} \underline{W}_{n}^{E}-\frac{1}{\mathrm{k}^{+}} \boldsymbol{\pi} \underline{F}_{n}^{E}
\end{gathered}
$$

Il est à noter que le traitement de la non linéarité de contact s'effectue au niveau de l'étape locale; elle ne modifie ainsi que peu le nombre d'itérations nécessaire à convergence. L'introduction de frottement dans le contact peut se faire de façon modulaire en envisageant un nouveau type d'interface correspondant à ce nouveau type de comportement, voir par exemple [COC 95], WRO 95].

- Étape linéaire : $\hat{\mathbf{s}}_{n+1 / 2} \rightarrow \mathbf{s}_{n+1}$, avec

$$
\mathbf{s}_{n+1} \in \mathbf{A}_{d}
$$

et vérifiant la direction de recherche $\left(\mathbf{s}_{n+1}-\hat{\mathbf{s}}_{n+1 / 2}\right) \in \mathbf{E}^{-}$qui se traduit par

$$
\left(\underline{F}_{n+1}^{E}-\underline{\hat{F}}_{n+1 / 2}^{E}\right)+\mathbf{k}^{-}\left(\underline{W}_{n+1}^{E}-\underline{\hat{W}}_{n+1 / 2}^{E}\right)=0
$$

en tout point des $\partial \Omega^{E}$. De la même façon, $\mathbf{k}^{-}$peut être pris égal à $\mathrm{k}^{-} \mathbb{I}_{d}$ où $\mathrm{k}^{-}$est un paramètre scalaire positif.

Tous les problèmes relatifs aux sous-structures $\Omega^{E}$ sont indépendants et peuvent donc être résolus en parallèle.

- Initialisation de l'algorithme :

La recherche d'un élément $\mathbf{s}_{0}$ admissible peut se faire de différentes manières. Une façon simple de procéder consiste à construire $\hat{\mathbf{s}}_{-1 / 2} \equiv 0$ qui a les propriétés voulues pour faire partie de $\boldsymbol{\Gamma}$, puis avec la direction de recherche $\mathbf{k}^{-}$, de redescendre sur $\mathbf{A}_{d}$.

\subsection{Quelques autres méthodes de sous-structuration}

La résolution du problème de l'assemblage de sous-structures se réalise généralement en considérant le problème condensé sur les interfaces. Les méthodes se classent alors en :

- méthodes directes, où un tel opérateur condensé - par exemple sur les degrés de liberté en déplacement de l'ensemble des interfaces - est explicitement construit ESC 94] de façon similaire à celle des macroéléments finis que sont les sous-structures; 
- méthodes itératives pour lesquelles :

- le déplacement reste continu aux interfaces $\left(\underline{W}^{E}=\underline{W}^{E^{\prime}}\right)$ et la discontinuité en effort est réduite au cours des itérations : par exemple la méthode de Schur primale pour laquelle le problème condensé est résolu par gradient conjugué [ROU 90], [DER 92];

- les efforts sont équilibrés aux interfaces $\left(\underline{F}^{E}+\underline{F}^{E^{\prime}}=0\right)$ et la discontinuité en déplacement est à réduire : c'est la méthode duale de la précédente [FAR 94b;

- ni les efforts, ni les déplacements n'ont à être continus au cours des itérations : ce sont alors des méthodes "mixtes" telles que l'approche que nous proposons et qui se confond dans le cas du comportement linéaire et d'interfaces parfaites avec [LIO 90] et [GLO 90, LAD 85.

De telles méthodes conduisent à chaque itération à résoudre un problème de structure sur chaque sous-structure et font intervenir des directions de recherche qui amèment à une plus ou moins grande synchronisation des processeurs (calcul d'une direction de recherche orthogonale pour le gradient conjugué par exemple).

\subsection{Convergence}

De par la nature du problème à traiter — le temps n'intervient pas-, l'espace $\mathbf{S}$ est équipé de la forme bilinéaire "travail" :

$$
\begin{aligned}
& \left(\mathbf{s}, \mathbf{s}^{\prime}\right) \\
& \mathbf{S} \times \mathbf{S}
\end{aligned} \rightarrow\left[\mathbf{s}, \mathbf{s}^{\prime}\right]
$$

avec

$\left[\mathbf{s}, \mathbf{s}^{\prime}\right]=\sum_{E \in \mathrm{E}} \frac{1}{2} \int_{\Omega^{E}}\left[\operatorname{Tr}\left(\boldsymbol{\sigma}^{E}{\boldsymbol{\varepsilon}^{\prime}}^{E}\right)+\operatorname{Tr}\left({\boldsymbol{\sigma}^{\prime}}^{E} \boldsymbol{\varepsilon}^{E}\right)\right] d \Omega-\frac{1}{2} \int_{\partial \Omega^{E}}\left(\underline{F}^{E} \cdot{\underline{W^{\prime}}}^{E}+{\underline{F^{\prime}}}^{E} \cdot \underline{W}^{E}\right) d S$

Les propriétés suivantes sont alors vérifiées dans le cas de liaisons parfaites ou de contacts unilatéraux sans frottement :

$$
\begin{aligned}
\forall\left(\hat{\mathbf{s}}, \hat{\mathbf{s}}^{\prime}\right) \in \boldsymbol{\Gamma} \times \boldsymbol{\Gamma} & {\left[\hat{\mathbf{s}}^{\prime}-\hat{\mathbf{s}}, \hat{\mathbf{s}}^{\prime}-\hat{\mathbf{s}}\right] \geq 0 } \\
\forall\left(\mathbf{s}, \mathbf{s}^{\prime}\right) \in \mathbf{A}_{d} \times \mathbf{A}_{d} & {\left[\mathbf{s}^{\prime}-\mathbf{s}, \mathbf{s}^{\prime}-\mathbf{s}\right] \leq 0 }
\end{aligned}
$$

Il est montré dans LAD 96] qu'elles permettent de conclure quant à la convergence de l'algorithme si les directions de recherches sont orthogonales au sens de la forme bilinéaire, ce qui se ramène à $\mathbf{k}^{+}=\mathbf{k}^{-}=\mathbf{k}$. Il y a convergence au sens où $\frac{1}{2}\left(\mathbf{s}_{n}+\mathbf{s}_{n+1}\right)$ tend vers la solution exacte $\mathbf{s}_{e x}$, tout en ayant

$$
\lim _{n \rightarrow \infty} \sum_{E \in \mathrm{E}} \int_{\Omega^{E}} \operatorname{Tr}\left(\boldsymbol{\sigma}_{n}^{E}-\boldsymbol{\sigma}_{e x}\right) \mathbf{K}\left(\boldsymbol{\sigma}_{n}^{E}-\boldsymbol{\sigma}_{e x}\right) d \Omega=0
$$


La convergence est assurée complètement si, de plus, il existe un point de l'assemblage en lequel le nombre de sous-structures attachées est impair, supérieur à 2 .

À fin d'illustration, prenons le cas unidimensionnel d'une poutre en traction soumise à deux déplacements imposés $\underline{U}_{d}^{A}$ et $\underline{U}_{d}^{B}$ en ses extrémités $A$ et $B$, et décomposée en 2 sous-structures $\Omega^{E}$ et $\Omega^{E^{\prime}}$ et 3 interfaces (dont 2 interfaces de déplacement imposé). La figure 4 explicite les notations.

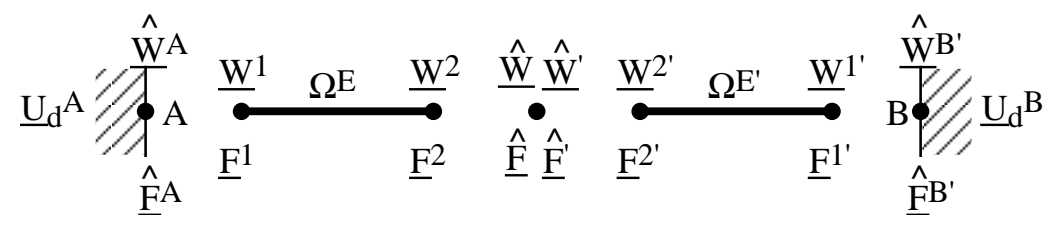

Figure 4: Exemple unidimensionnel

Supposons connu $\mathbf{s}_{n}$ de $\mathbf{A}_{d}$; l'admissibilité se traduit, par exemple sur la sous-structure $\Omega^{E}$ dont la raideur est notée $\mu$, par :

$$
\left[\begin{array}{l}
\underline{F}_{n}^{1} \\
\underline{F}_{n}^{2}
\end{array}\right]=[K]\left[\begin{array}{l}
\underline{W}_{n}^{1} \\
\underline{W}_{n}^{2}
\end{array}\right] \quad \text { où } \quad[K]=\mu\left[\begin{array}{rr}
1 & -1 \\
-1 & 1
\end{array}\right]
$$

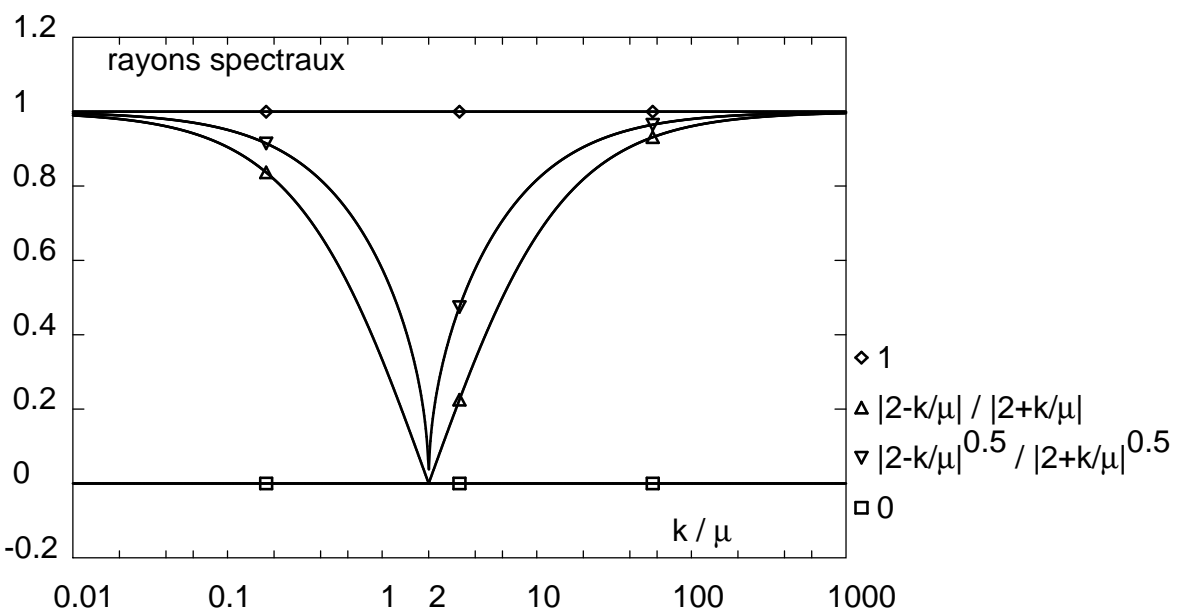

FiguRE 5: Rayons spectraux fonctions du paramètre $k$ 
L'étape locale permet d'écrire :

$$
\begin{aligned}
\hat{\underline{F}}_{n+1 / 2}^{A}+k \underline{\hat{W}}_{n+1 / 2}^{A} & =\underline{F}_{n}^{1}-k \underline{W}_{n}^{1}+2 k \underline{U}_{d}^{A}, \\
\underline{\hat{E}}_{n+1 / 2}+k \underline{\hat{W}}_{n+1 / 2} & =-\underline{F}_{n}^{2^{\prime}}+k \underline{W}_{n}^{2^{\prime}}, \\
\hat{\hat{E}}_{n+1 / 2}^{B^{\prime}}+k \underline{\hat{W}}_{n+1 / 2}^{B^{\prime}} & =\underline{F}_{n}^{1^{\prime}}-k \underline{W}_{n}^{1^{\prime}}+2 k \underline{U}_{d}^{B}, \\
\underline{\hat{F}}_{n+1 / 2}^{\prime}+k \underline{\hat{W}}_{n+1 / 2}^{\prime} & =\underline{F}_{n}^{1^{\prime}}+k \underline{W}_{n}^{1^{\prime}}
\end{aligned}
$$

L'étape globale suivante permet ainsi de trouver $\mathbf{s}_{n+1}$. Par exemple, pour $\Omega^{E}$ :

$([K]+[k])\left[\begin{array}{l}\underline{W}_{n+1}^{1} \\ \underline{W}_{n+1}^{2}\end{array}\right]=\left[\begin{array}{l}\hat{F}_{n+1 / 2}^{A}+k \underline{\hat{W}}_{n+1 / 2}^{A} \\ \underline{\hat{F}}_{n+1 / 2}+k \underline{\hat{W}}_{n+1 / 2}\end{array}\right] \quad$ où $\quad[k]=k\left[\begin{array}{ll}1 & 0 \\ 0 & 1\end{array}\right]$ traduit la direction de recherche.

Finalement, $\mathbf{s}_{n+1}$ et $\mathbf{s}_{n}$ sont liés de façon affine par : $\left[C_{n+1}\right]=[A]\left[C_{n}\right]+$ $\left[B_{d}\right]$

$$
\operatorname{avec}\left[C_{n}\right]=\left[\begin{array}{c}
k \underline{W}_{n}^{1} \\
k \underline{W}_{n}^{2} \\
\underline{F}_{n}^{1} \\
\underline{F}_{n}^{2} \\
k \underline{W}_{n}^{2^{\prime}} \\
k \underline{W}_{n}^{1^{\prime}} \\
\underline{F}_{n}^{2^{\prime}} \\
\underline{F}_{n}^{1^{\prime}}
\end{array}\right],[A]=\frac{1}{k+2 \mu}\left[\begin{array}{cccccccc}
-\nu & 0 & \nu & 0 & \mu & 0 & -\mu & 0 \\
-\mu & 0 & \mu & 0 & \nu & 0 & -\nu & 0 \\
-\mu & 0 & \mu & 0 & -\mu & 0 & \mu & 0 \\
\mu & 0 & -\mu & 0 & \mu & 0 & -\mu & 0 \\
0 & \nu & 0 & -\nu & 0 & -\mu & 0 & \mu \\
0 & \mu & 0 & -\mu & 0 & -\nu & 0 & \nu \\
0 & \mu & 0 & -\mu & 0 & \mu & 0 & -\mu \\
0 & -\mu & 0 & \mu & 0 & -\mu & 0 & \mu
\end{array}\right]
$$

et $\nu=k+\mu$, et où $\left[B_{d}\right]$ ne dépend que du chargement donné. La convergence serait alors assurée si tous les modules des valeurs propres de $[A]$ étaient strictement inférieurs à 1 . Ici, les valeurs propres $\lambda_{i}$ de $[A]$ sont

$$
\left(-1,0,0,0,0, \frac{2 \mu-k}{2 \mu+k}, \pm \sqrt{\frac{k-2 \mu}{k+2 \mu}}\right)
$$

et leurs modules sont tracés sur la figure 5 en fonction du choix du paramètre $k$. On note ainsi que la valeur propre - 1 correspond à un déplacement de solide rigide par sous-structure, ce qui illustre la convergence partielle pré-citée. Si on note $\left[C_{e x}\right]$ la colonne associée à la solution exacte $\mathbf{s}_{e x}$,

$$
\left[C_{e x}\right]=[A]\left[C_{e x}\right]+\left[B_{d}\right] \quad \text { et }\left(\left[C_{n+1}\right]-\left[C_{e x}\right]\right)=[A]\left(\left[C_{n}\right]-\left[C_{e x}\right]\right)
$$

la colonne associée à $\frac{1}{2}\left(\mathbf{s}_{n+1}+\mathbf{s}_{n}\right)-\mathbf{s}_{e x}$ est alors

$$
\begin{aligned}
\frac{1}{2}\left(\left[C_{n+1}\right]-\left[C_{e x}\right]+\left[C_{n}\right]-\left[C_{e x}\right]\right) & =[A]^{n-1} \frac{1}{2}\left(\left[C_{2}\right]-\left[C_{e x}\right]+\left[C_{1}\right]-\left[C_{e x}\right]\right) \\
& =[A]^{n-1} \frac{1}{2}\left([A]+\mathbb{I}_{d}\right)\left(\left[C_{1}\right]-\left[C_{e x}\right]\right)
\end{aligned}
$$

Les valeurs propres de $[A]^{n-1} \frac{1}{2}\left([A]+\mathbb{I}_{d}\right)$ étant alors les $\frac{1}{2}\left(1+\lambda_{i}\right) \lambda_{i}^{n-1}$, toutes de module strictement inférieur à 1 , on a donc bien convergence de 
$\frac{1}{2}\left(\mathbf{s}_{n+1}+\mathbf{s}_{n}\right)$ vers $\mathbf{s}_{e x}$. Il apparaît de plus une valeur optimale de $k$ égale à $2 \mu$ sur cet exemple.

De façon générale, on montre également que la quantité :

$$
\begin{array}{r}
\left\|\hat{\mathbf{s}}_{n+1 / 2}-\mathbf{s}_{n}\right\|=\frac{1}{2}\left\{\sum _ { E \in \mathrm { E } } \int _ { \partial \Omega ^ { E } } \left[\left(\underline{\hat{F}}_{n+1 / 2}^{E}-\underline{F}_{n}^{E}\right) \cdot \mathbf{k}^{-1}\left(\underline{\hat{F}}_{n+1 / 2}^{E}-\underline{F}_{n}^{E}\right)+\right.\right. \\
\left.\left.+\left(\underline{\hat{W}}_{n+1 / 2}^{E}-\underline{W}_{n}^{E}\right) \cdot \mathbf{k}\left(\underline{\hat{W}}_{n+1 / 2}^{E}-\underline{W}_{n}^{E}\right)\right] d S\right\}^{\frac{1}{2}}
\end{array}
$$

est une fonction décroissante de $n$ qui tend vers 0 avec $1 / n$. Elle peut donc être utilisée pour bâtir des indicateurs d'erreur.

\section{Premières mises en œuvre et comportement de l'algorithme}

\subsection{Mise en œuvre numérique}

Pour réaliser l'implantation de l'algorithme, une approche éléments finis a été utilisée [IE 91].

Il est aisé de voir que l'étape linéaire se ramène à la résolution d'un problème de type élasticité sur la sous-structure $\Omega^{E}$; la formulation en déplacement correspondant est :

$$
\begin{array}{r}
\min _{\underline{U}^{E} \in \mathcal{U}_{a d}^{E}, \underline{U}^{E}}{ }_{\mid \partial \Omega^{E}}=\underline{W}^{E} \quad \frac{1}{2} \int_{\Omega^{E}} \operatorname{Tr}\left[\varepsilon\left(\underline{U}^{E}\right) \mathbf{K} \varepsilon\left(\underline{U}^{E}\right)\right] d \Omega+ \\
+\frac{1}{2} \int_{\partial \Omega^{E}}\left(\underline{W}^{E}-\underline{\hat{W}}_{n+1 / 2}^{E}\right) \cdot \mathbf{k}^{-}\left(\underline{W}^{E}-\underline{\hat{W}}_{n+1 / 2}^{E}\right) d S-\int_{\partial \Omega^{E}} \underline{\hat{F}}_{n+1 / 2}^{E} \cdot \underline{W}^{E} d S
\end{array}
$$

c'est à dire trouver $\underline{U}^{E}$ de $\mathcal{U}_{a d}^{E}$ tel que

$$
\begin{array}{r}
\forall \underline{U}^{\star} \in \mathcal{U}_{a d}^{E}, \int_{\Omega^{E}} \operatorname{Tr}\left[\varepsilon\left(\underline{U}^{E}\right) \mathbf{K} \varepsilon\left(\underline{U}^{\star}\right)\right] d \Omega+\int_{\partial \Omega^{E}} \underline{U}^{E} \cdot \mathbf{k}^{-} \underline{U}^{\star} d S= \\
=\int_{\Omega^{E}} \underline{f}_{d} \cdot \underline{U}^{\star} d \Omega+\int_{\partial \Omega^{E}}\left(\underline{\hat{F}}_{n+1 / 2}^{E}+\mathbf{k}^{-} \underline{\hat{W}}_{n+1 / 2}^{E}\right) \cdot \underline{U}^{\star} d S
\end{array}
$$

Une fois discrétisée, elle conduit à un système de la forme :

$$
\left(\left[K^{E}\right]+\left[k^{E}\right]\right)\left[q^{E}\right]=\left[f^{E}\right]
$$

où $\left[K^{E}\right]$ est la matrice de rigidité de la sous-structure, $\left[k^{E}\right]$ une rigidité "bord", toutes deux constantes au cours des itérations. La factorisation de Cholesky de $\left(\left[K^{E}\right]+\left[k^{E}\right]\right)$, qui est stockée "bande", est réalisée une fois pour toute lors de la phase d'initialisation. Le maillage de chaque sous-structure $\Omega^{E}$ — choisi ici compatible entre celles-ci- traduit la discrétisation adoptée pour le déplacement $\underline{U}_{n+1}^{E}$. Celle du déplacement bord $\underline{W}_{n+1}^{E}$ s'en déduit 
par restriction sur $\partial \Omega^{E}$. En post-traitement, les efforts bord sont obtenus à partir de la direction de recherche de l'étape globale :

$$
\underline{F}_{n+1}^{E}=\underline{\hat{F}}_{n+1 / 2}^{E}+\mathbf{k}^{-} \underline{\hat{W}}_{n+1 / 2}^{E}-\mathbf{k}^{-} \underline{W}_{n+1}^{E} \quad \text { sur } \partial \Omega^{E}
$$

c'est dire que leur discrétisation est identique à celle du déplacement bord, pour peu que $\underline{\hat{F}}^{E}$ et $\underline{\hat{W}}^{E}$ restent réguliers, comme c'est le cas pour une interface parfaite. L'algorithme présenté converge alors vers la solution éléments finis directe sur l'ensemble de la structure. Dans d'autres cas, comme le contact unilatéral par exemple, on peut pour $\left(\hat{W}^{E} ; \hat{F}^{E}\right)$ imposer la même discrétisation que celle de $\left(\underline{W}^{E} ; \underline{F}^{E}\right)$. D'autres discrétisations mieux adaptées peuvent néanmoins être envisagées.

En prenant $\mathbf{k}^{-}=\mathbf{k}^{+}=\mathbf{k}=\frac{E}{L_{0}} \mathbb{I}_{d}$ (où $E$ désigne le module d'Young du matériau et $\mathbb{I}_{d}$, l'opérateur identité), rechercher une valeur optimale pour les directions de recherche revient à trouver une longueur caractéristique $L_{0}$ optimale. Cette longueur existe et est, pour des structures massives, voisine de la plus grande dimension de la structure étudiée. Lorsque la structure est élancée, et que les effets de flexion sont importants, il est intéressant de prendre, pour $L_{0}$ une valeur plus importante. Le nombre d'itérations pour atteindre la convergence pratique est, pour ce dernier type de problème, plus important que pour des structures massives.

\subsection{Comportement de la méthode - Comparaison avec une méthode directe}

Divers paramètres influent sur le comportement de la méthode, ou plus précisément, sur le nombre d'itérations nécessaires pour atteindre la convergence pratique de l'algorithme. Parmi ceux-ci, on trouve plus particulièrement : la direction de recherche $\mathbf{k}$, le nombre de sous-structures dans une direction donnée et la nature du problème (forme de la structure et nature de la décomposition en sous-structures). En particulier, une décomposition "homogène" (sous-structures massives plutôt qu'élancées) donne en général de bons résultats.

Afin d'estimer les performances de l'approche proposée, nous avons comparé le nombre total d'opérations en virgule flottante qu'elle nécessite, pour résoudre un problème incluant une décomposition de domaine, avec le nombre d'opérations en virgule flottante que nécessite une méthode directe de type Crout pour résoudre le même problème mais sans décomposition. Ce décompte d'opérations comprend pour l'initialisation, celles nécessaires à la factorisation de Crout des matrices $\left(\left[K^{E}\right]+\left[k^{E}\right]\right)$, ainsi que pour toutes les itérations, celles nécessaires lors des étapes globales au calcul des seconds membres $\left[f^{E}\right]$, aux montées-descentes, aux projections des $\left[q^{E}\right]$ sur $\partial \Omega^{E}$ en $\underline{W}^{E}$ et au calcul des $\underline{F}^{E}$, et enfin lors des étapes locales, aux calculs $\operatorname{des} \underline{\hat{W}}^{E}$ et $\underline{\hat{F}}^{E}$. 
Nous introduisons alors le rapport $G$ entre le nombre d'opérations utilisées par la méthode directe, où la matrice factorisée est aussi stockée "bande" et le nombre de celles nécessaires à la convergence pour l'assemblage de sous-structures. Ce rapport dépend essentiellement, pour un problème donné, des paramètres suivants :

- nombre de degrés de liberté de la discrétisation,

- nombre de sous-structures crées lors de la décomposition du domaine,

- nombre d'itérations nécessaire à la convergence "pratique".

Nous considérons que la convergence "pratique" est atteinte lorsque l'erreur en énergie, calculée vis à vis de la solution obtenue par la méthode directe qui est la solution éléments finis du problème sans décomposition de domaine, est inférieure à $1 \%$.

Sur une poutre en flexion traitée en élasticité plane, de module d'Young $E=2.10^{5} \mathrm{MPa}$, de coefficient de Poisson $\nu=0,35$, et dont le rapport longueur $L$ sur hauteur est de 3 (figure 6), des gains commencent à être observés pour des discrétisations relativement grossières (5000 degrés de liberté, éléments triangulaires à 6 nœuds) (figure 7). Ces gains sont d'autant plus importants que la discrétisation est fine. Il existe, de plus, un optimum pour le choix de la décomposition du domaine en fonction du nombre de degrés de liberté du problème à traiter. Pour un problème à 33000 degrés de liberté, cet optimum correspond à une décomposition en 16 sous-structures $(G=1,7)$ et pour un problème à 500000 degrés de liberté à une décomposition en 64 sous-structures $(G=9,8)$.

Pour le tracé de la figure 7, nous avons fait l'hypothèse que le nombre d'itérations à convergence pratique, pour un problème et une décomposition en sous-structures donnés, ne dépend que peu du raffinement du maillage. Dans l'exemple précédent et pour un problème de référence de 33282 degrés de liberté, ce nombre d'itérations est reporté dans la table 1 suivant le nombre de sous-structures choisi. On illustre ici le cas de convergence difficile pour les structures élancées travaillant en flexion puisque lorsque l'on dispose de 32 sous-structures dans la longueur de la poutre, plus de 1000 itérations sont nécessaires. La table 2, quant à elle, montre pour ce même problème avec $4 \times 4$ sous-structures, l'influence du choix de la direction de recherche $\mathbf{k}=\frac{E}{L_{0}} \mathbb{I}_{d}$. De plus, le rapport $R$ entre le nombre d'opérations de la phase de factorisation et le nombre total d'opérations dans un tel cas d'élasticité plane est illustré pour le même exemple par la figure 8

\begin{tabular}{|c|cccccc|}
\hline nb. de sous-structures & 1 & $2 \times 2$ & $4 \times 4$ & $8 \times 8$ & $16 \times 16$ & $32 \times 32$ \\
\hline nb. d'itérations & 1 & 39 & 128 & 297 & 630 & 1296 \\
\hline
\end{tabular}

TABLE 1: Influence du nombre de sous-structures sur le nombre d'itérations nécessaire à la convergence pratique 

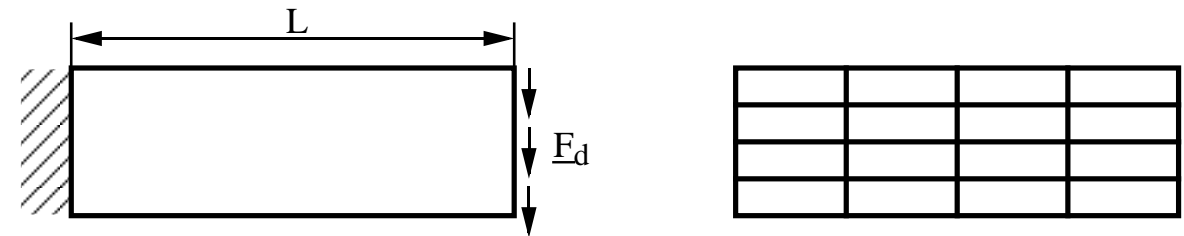

Figure 6: Problème test - poutre en flexion, élasticité plane et exemple de décomposition en $4 \times 4$ sous-structures

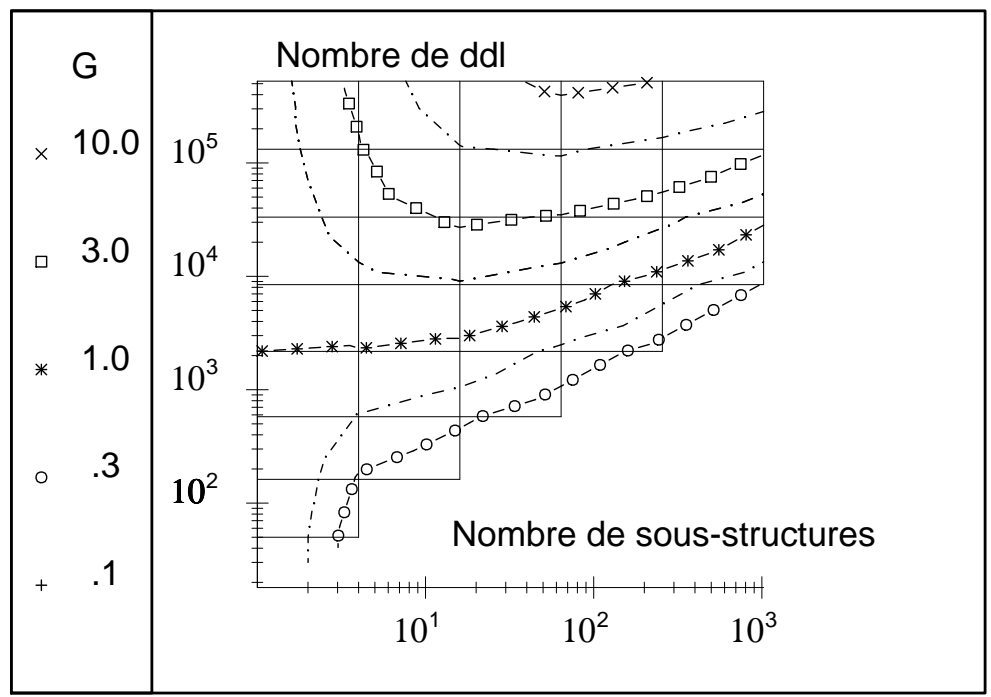

FiguRE 7: Isovaleurs du rapport $G$ des nombres d'opérations méthode directe et méthode proposée

\begin{tabular}{|l|ccccccc|}
\hline rapport $\frac{L_{0}}{L}$ & 2 & 2,5 & 3 & 3,5 & 4 & 5 & 6 \\
\hline nb. d'itérations & 208 & 157 & 128 & 134 & 139 & 162 & 202 \\
\hline
\end{tabular}

TABLE 2: Influence de la direction de recherche $\mathbf{k}=\frac{E}{L_{0}} \mathbb{I}_{d}$ sur le nombre d'itérations nécessaire à la convergence pratique pour $4 \times 4$ sous-structures 


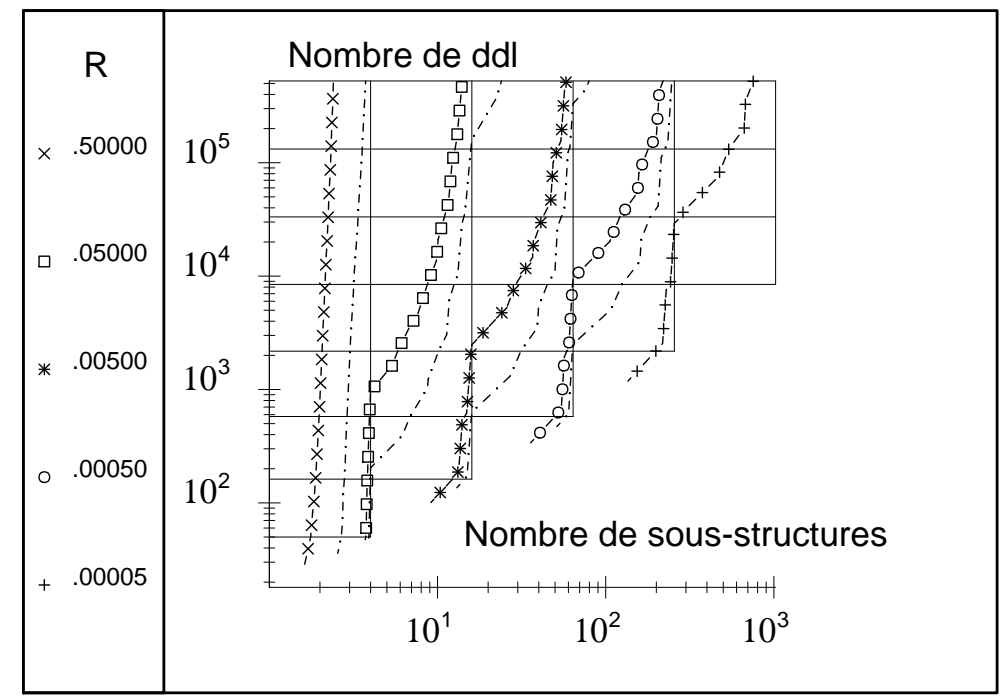

FiguRE 8: Isovaleurs du rapport $R$ du nombre d'opérations pour la factorisation de toutes les sous-structures au nombre total d'opérations jusqu'à convergence

Cette dernière remarque montre que notre approche est plutôt adaptée à des décompositions de domaine comprenant un nombre réduit de sousstructures (jusqu'à une centaine sur cet exemple bidimensionnel). Lorsque le nombre de sous-structures augmente le comportement de l'algorithme se dégrade et l'utilisation de stratégies multi-échelles devrait améliorer considérablement celui-ci YYSE 86, [BRA 86].

\subsection{Comportement sur calculateurs parallèles}

L'algorithme a été dans un premier temps implanté sur deux types de calculateurs parallèles :

- calculateur MIMD à mémoire partagée (ALLIANT 2800),

- calculateur MIDM à mémoire distribuée (iPSC i860 d'Intel et nCUBE2).

Les performances "parallèles" obtenues ont été estimées à partir d'une efficacité CAR 91 définie comme étant le rapport entre le temps moyen $T_{\text {moy }}$ pris par les processeurs pour les seuls traitements numériques et la durée totale de l'exécution de l'algorithme $T_{N}$ ( $N$ étant le nombre de processeurs utilisés). La décomposition de la structure en sous-structures fait partie du "pré-processing" et n'est pas prise en compte dans cette partie. Sur machines à mémoire partagée (ALLIANT), le temps moyen est calculé à partir du temps $T_{1}$ pris par l'execution du problème sur un seul processeur (donc sans pertes liées au parallélisme), divisé par le nombre total de 
processeurs. L'efficacité $e_{M P}$ s'écrit alors :

$$
e_{M P}=\frac{T_{1} / N}{T_{N}}=\frac{T_{1}}{T_{N}} \frac{1}{N}=\operatorname{gain} \frac{1}{N}
$$

Le gain (ou "speed up") est la grandeur classiquement utilisée sur ce type de machine. Sur machines à mémoire distribuée (iPCS ou nCUBE2), le temps moyen est la moyenne des temps $T_{C a l}^{i}$ pris par chaque processeur pour les seuls traitements numériques. Les mesures de temps sont effectuées à partir de prises de temps horloges. Il est en effet aisé de dissocier les zones du programme dédiées aux calculs de celles dédiées à l'envoi ou à la réception de messages. L'efficacité sur ce type de calculateurs s'écrit alors :

$$
e_{M D}=\frac{\frac{1}{N} \sum_{i=1}^{N} T_{C a l}^{i}}{T_{N}}
$$

L'étude du comportement de l'algorithme sur ALLIANT confirme les limitations dues au partage de la mémoire et en particulier à la taille de la mémoire cache. Ce type de machine (avec cache) est généralement mieux adapté au microtasking, c'est à dire à un parallélisme portant sur des boucles où les traitements à effectuer sont réduits et portent sur un nombre restreint de données, qu'au macrotasking (parallélisme sur des tâches importantes). Dans notre application, le parallélisme est effectué au niveau des sous-structures et porte donc sur un jeu de données important par processeur, jeu de données qui déborde largement de l'espace disponible sur le cache. Lorsque l'exécution du programme a lieu sur un seul processeur, celui-ci dispose de tout le cache; alors que lorsque plusieurs processeurs sont utilisés, ceux-ci se partagent le cache, ce qui accentue les problèmes de débordement. Ainsi, sur ALLIANT FX2800, avec une utilisation des 10 processeurs disponibles, l'efficacité mesurée ne dépasse pas $50 \%$ pour des sous-structures comprenant 1922 degrés de liberté chacune (table 3).

\begin{tabular}{|c|c|c|c|c|}
\hline Nombre de processeurs & 1 & 2 & 5 & 10 \\
\hline Factorisation (s) & 30 & 15,8 & 6,75 & 4,9 \\
\hline Efficacité & $100 \%$ & $95 \%$ & $88,9 \%$ & $61,2 \%$ \\
\hline 10 itérations (s) & 46 & 29 & 13,9 & 9,4 \\
\hline Efficacité & $100 \%$ & $79,3 \%$ & $66,2 \%$ & $48,9 \%$ \\
\hline
\end{tabular}

TABLE 3: Mesures d'efficacité sur ALLIANT FX2800

Les expérimentations menées sur iPSC, avec 128 processeurs, et sur nCUBE, avec 64 processeurs, montrent la bonne aptitude de l'algorithme, comme c'est le cas des méthodes de décomposition de domaine, à fonctionner sur ce type de machine. Les efficacités obtenues sont supérieures à $97 \%$ 
pour des problèmes occupant plus de $25 \%$ de l'espace mémoire disponible. Même pour des problèmes de taille modeste (3\% de l'espace mémoire disponible), l'efficacité reste supérieure à $70 \%$. Ces résultats s'entendent pour une répartition parfaitement équitable des charges de travail par processeur. En fait, les pertes liées au parallélisme sont essentiellement dûes, sur ce type de calculateurs, aux déséquilibres des tâches entre les processeurs, et plus précisément, aux attentes dans les phases de synchronisation des processeurs les moins chargés.

\section{Exemple de problème tridimensionnel}

Afin d'illustrer la faisabilité de l'approche sur des calculs tridimensionnels, l'algorithme a été implanté dans le cadre de l'atelier logiciel CASTEM2000 VER 88. L'exemple proposé ici figure 9 concerne un couple de dents d'engrenage pour lequel pignon et crémaillère présentent un défaut angulaire de positionnement. Le module de taille est de l'ordre de 1,5 mm, et $\left\|\underline{U}_{d}\right\|=0,02 \mathrm{~mm}$. Le contact entre ces pièces est supposé sans frottement.
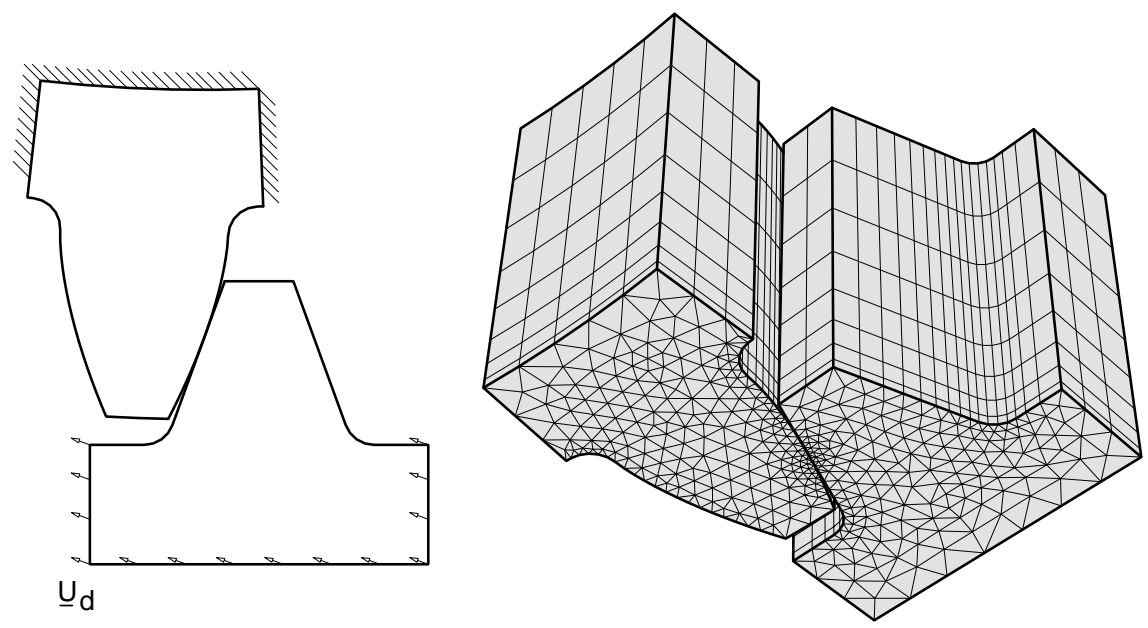

Figure 9: Exemple traité

Le matériau est caractérisé par un module d'Young $E=2.10^{5} \mathrm{MPa}$ et un coefficient de Poisson $\nu=0,3$. Pour traiter ce problème, une décomposition automatique en 64 sous-structures a été effectuée (figure 10, les interfaces traitant des liaisons parfaites ainsi que des contacts unilatéraux sans frottement.

Ces sous-structures et interfaces ont ensuite été distribuées sur les 64 processeurs du nCUBE2S. Sur la figure 11 sont représentés les champs de contrainte de Von Misès obtenus à l'itération 1 et à convergence à l'itération 


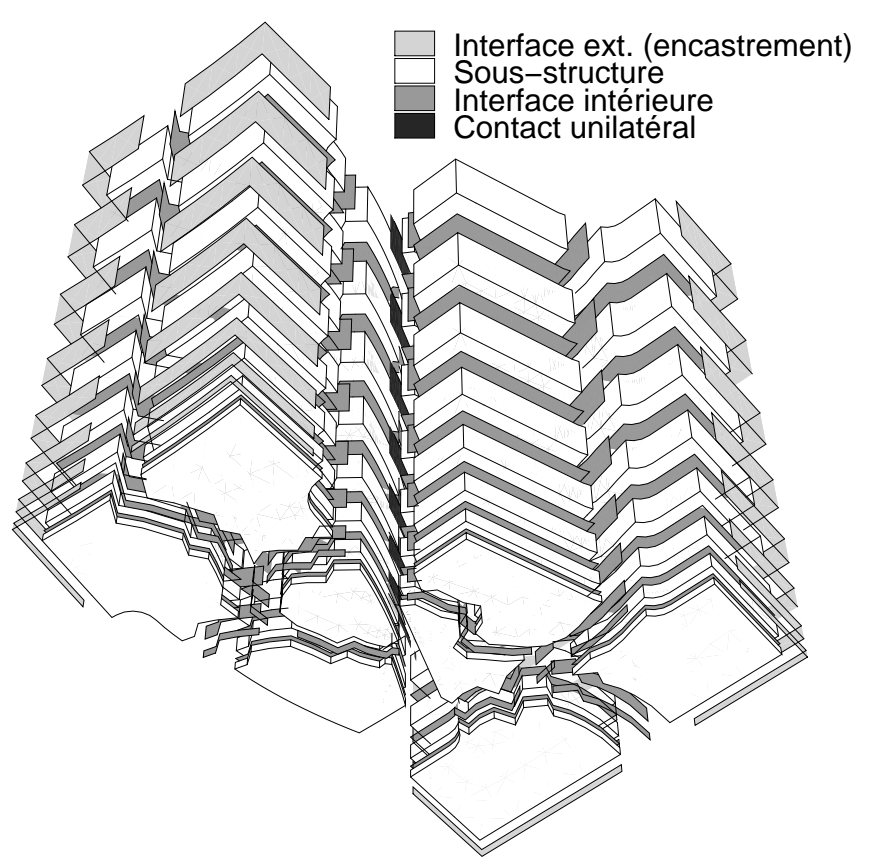

Figure 10: Décomposition 
40 , c'est à dire pour $\mathbf{s}_{1}$ et $\mathbf{s}_{40}$. L'évolution de l'indicateur d'erreur

$$
\frac{\left\|\hat{\mathbf{s}}_{n+1 / 2}-\mathbf{s}_{n}\right\|}{\left\|\hat{\mathbf{s}}_{n+1 / 2}\right\|+\left\|\mathbf{s}_{n}\right\|}
$$

est tracée sur la figure 12 La longueur $L_{0}$ caractérisant la direction de recherche $\mathbf{k}=\frac{E}{L_{0}} \mathbb{I}_{d}$ y est aussi représentée. Sur cet exemple, où le nombre maximum de sous-structures dans une direction donnée est de l'ordre de 8 , une erreur de $0.5 \%$ est obtenue en une quarantaine d'itérations.

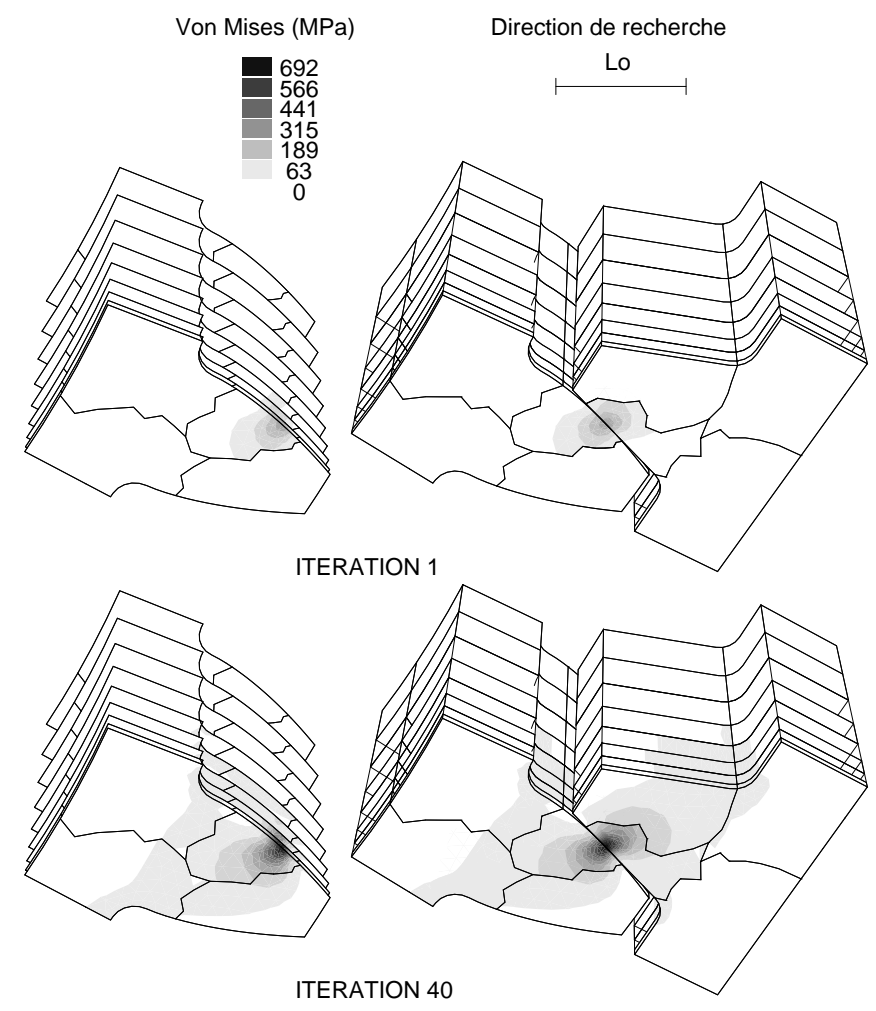

FiguRE 11: Résultats obtenus

Chaque processeur doit alors traiter un problème dont la taille moyenne est de $370 \mathrm{ddl}$ (encombrement moyen de la matrice de rigidité $\left[K^{E}\right]+[k]^{E}$ de $125 \mathrm{Ko}$, soit $8 \mathrm{Mo}$ au total). Le calcul séquentiel correspondant possède 11600 ddl (encombrement de 35 Mo). Le temps horloge (ou "wall-clock") total de traitement pour cet exemple est de $120 \mathrm{~s}$. Il se partage en $45 \mathrm{~s}$ pour la phase d'initialisation et $75 \mathrm{~s}$ pour réaliser 40 itérations; la puissance effective mesurée sur 1 processeur étant de 1 Mflops. Sur ces mêmes prises de 


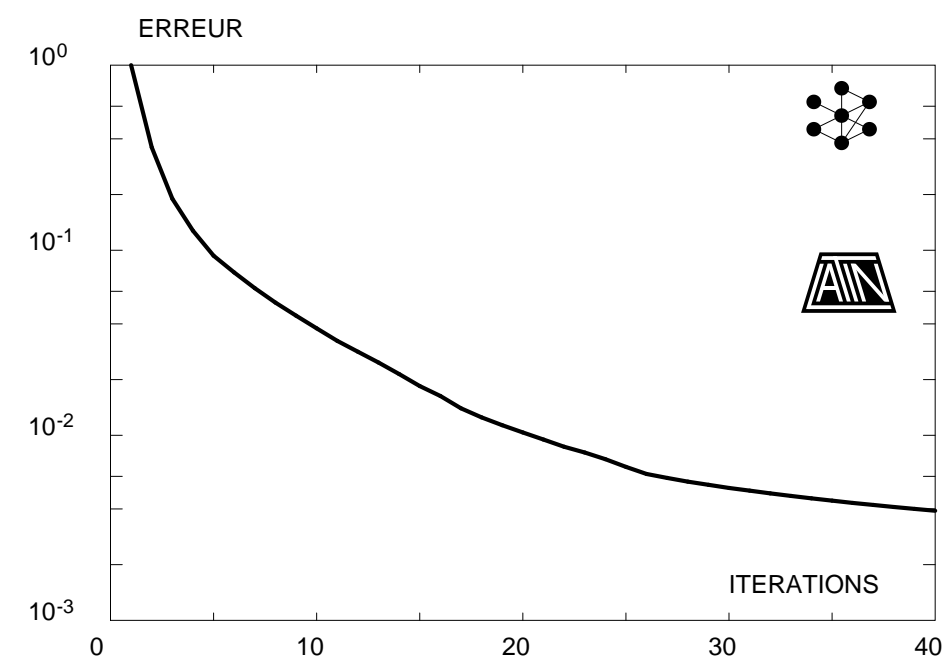

FiguRE 12: Indicateur d'erreur en fonction du nombre d'itérations

temps, l'efficacité obtenue est de $70 \%$; les pertes de parallélisme sont ici dûes d'une part à l'accès disque initial qui désynchronise la phase d'initialisation puisque les 64 processeurs chargent leurs données à partir d'un seul disque, et d'autre part à un déséquilibre des charges entre processeurs dû à un découpage en sous-structures automatique dont le seul critère est un égal nombre d'éléments par sous-structure (une mesure de ce déséquilibre peut être illustrée par l'écart maximal entre encombrements des matrices de rigidité assemblées de chaque sous-structure, qui vaut $15 \%$ de la valeur moyenne).

\section{Conclusion}

La méthode de décomposition de domaine présentée ici comporte une double originalité. Elle présente le problème à étudier comme étant un assemblage d'entités, toutes mécaniques, les sous-structures et les interfaces, et elle utilise une approche à grand incrément de temps pour construire un algorithme de résolution itératif.

Les résultats présentés portent d'une part sur une première version de cette méthode de décomposition de domaine, dans le cadre de l'élasticité plane linéaire, et d'autre part, sur une implantation dans un code de calcul de type industriel pour des problèmes d'élasticité tridimensionnels.

Ces travaux, qui comportent des choix simples de description des champs propres aux sous-structures et aux interfaces à l'aide d'une approche éléments finis, ont permis d'une part, de cerner le comportement algorithmique de 
cette approche et de calibrer certains paramètres qui lui sont propres, mais aussi de mieux appréhender l'adaptabilité de la méthode de décomposition de domaine aux classes de calculateurs parallèles les plus répandues. Les expérimentations ont, de plus, montré que, même en séquentiel, une approche de décomposition de domaine peut s'avérer moins onéreuse (en nombre d'opérations en virgule flottante ainsi qu'en stockage de données) qu'une méthode directe de type Crout.

\section{Références}

[BRA 86] Bramble J. H., Pasciak J. E. et Schatz A. H., The construction of preconditioners for elliptic problems by substructuring, I, Math. Comp., vol. 47, n 175, 1986, pp. 103-134.

[BUO 93] Buoni J.J., Farrell P.A. et Ruttan A., Algorithms for LU decomposition on a shared memory multiprocessor, Parallel Computing, vol. 19, 1993, pp. 925-937.

[CAR 91] Carmona E. A. et Rice M. D., Modeling the serial and parallel fractions of a parallel algorithm, Journal of Parallel and Distributed Computing, n 13, 1991, pp. 286-298.

[CHA 90] Chan T., Glowinski R., Périaux J. et Windlun O. (édité par), Domain Decomposition Methods for Partial Differential Equations, SIAM, Philadelphie, USA, 1990.

[COC 95] Cocu M., Pratt E. et Raous M., Analysis of an incremental formulation for frictional contact problems, In Raous et al. RAO 95, pp. 13-20.

[DÉB 90] Débordes O. et Michel J.C., Parallélisation des problèmes nonlinéaires, Calcul des Structures et Intelligence Artificielle, éd. par Fouet J-M., Ladevèze P. et Ohayon R., pp. 223-232, Pluralis, 1990.

[DER 92] de Roeck Y.-H., Le Tallec P. et Vidrascu M., A domain decomposed solver for nonlinear elasticity, Comp. Meth. Appl. Mech. Engng., $\mathrm{n}^{\circ}$ 99, 1992, pp. 187-207.

[ESC 94] Escaig Y., Vayssade M. et Touzot G., Une méthode de décomposition de domaine multifrontale multiniveaux, Revue Européenne des Éléments Finis, vol. 3, 1994, pp. 311-337.

[FAR 88] Farhat C., A simple and efficient automatic fem domain decomposer, Computers \& Structures, vol. 28, n 5, 1988, pp. 579-602.

[FAR 91] Farhat C. et Roux F.-X., A method of finite element tearing and interconnecting and its parallel solution algorithm, Int. J. Numer. Meth. Engng., vol. 32, 1991, pp. 1205-1227. 
[FAR 94a] Farhat C., Mandel J. et Roux F.-X., Optimal properties of the FETI domain decomposition method, Comp. Meth. Appl. Mech. Engng., vol. 115, 1994, pp. 365-385.

[FAR 94b] Farhat C. et Roux F.-X., Implicit parallel processing in structural mechanics, Computational Mechanics Advances, éd. par Oden J. Tinsley, North-Holland, Juin 1994.

[GLO 90] Glowinski R. et Le Tallec P., Augmented lagrangian interpretation of the nonoverlapping schwarz alternating method, In Chan et al. CHA 90, pp. 224-231.

[LAD 85] Ladevèze J., Algorithmes adaptés aux calculs vectoriel et parallèle pour des méthodes de décomposition de domaines, Actes du 3ème colloque Tendances actuelles en calcul de structures, pp. 893-907, Bastia, Novembre 1985.

[LAD 92a] Ladevèze P., New advances in large time increment method, New advances in computational structural mechanics, éd. par Ladevèze P. et Zienkiewicz O. C., pp. 1-18, Elsevier, 1992.

[LAD 92b] Ladevèze P. et Lorong Ph., A large time increment approach with domain decomposition technique for mechanical non linear problems, Comp. Meths. Appl. Mech. Engrg., éd. par INRIA, pp. 569-578, 1992.

[LAD 93] Ladevèze P. et Lorong Ph., Formulations et stratégies parallèles pour l'analyse non linéaire des structures, Colloque national en calcul des structures, pp. 910-919, Giens, Mai 1993.

[LAD 96] Ladevèze P., Mécanique non-linéaire des structures - Nouvelle approche et méthodes de calcul non incrémentales, Paris, Hermès, 1996.

[LIO 90] Lions P.L., On the schwarz alternating method III. A variant for nonoverlapping subdomains, In Chan et al. [CHA 90].

[NOO 94] Noor A.K., New computing systems, future high performance computing environments and their implications on large-scale problems, Advances in parallel and vector processing for structural mechanics, éd. par Topping B.H.V. et Papadrakakis M., pp. 1-9, Edinbourg, Scotland, 1994.

[PAD 91] Padovan J. et Kwang A., Hierarchically parallelized constrained nonlinear solvers with automated substructuring, Computers $\&$ Structures, vol. 41, n 1,1991 , pp. 7-33.

[PAN 93] Pan V. et Reif J., Fast and efficient parallel solution of sparse linear systems, SIAM J. Sci. Comput., vol. 22, n 6, 1993, pp. $1227-1250$.

[RAO 95] Raous M., Jean M. et Moreau J.-J. (édité par), Proceedings of the Second Contact Mechanics International Symposium, New York, Plenum Press, 1995. 
[ROU 90] Roux F.-X., Méthodes de résolution par sous-domaines en statique, La Recherche Aérospatiale, $\mathrm{n}^{\circ}$ 1, 1990, pp. 37-48.

[VER 88] Verpeaux P., Charras T. et Millard A., CASTEM 2000 : une approche moderne du calcul des structures, Calcul des Structures et Intelligence Artificielle, éd. par Fouet J-M., Ladevèze P. et Ohayon R., pp. 261-271, Pluralis, 1988.

[WRO 95] Wronski M. et Jean M., Some computational aspects of structural dynamics problems with frictional contact, In Raous et al. RAO 95, pp. 137-144.

[YAG 93] Yagawa G., Yoshioka A., Yoshimura S. et Soneda N., A parallel finite element method with a supercomputer network, Computers $\& 3$ Structures, vol. 47, 1993, pp. 407-418.

[YSE 86] Yserentant H., On the multi-level splitting of finite element spaces, Num. Math., vol. 49, 1986, pp. 379-412.

[ZIE 91] Zienkiewicz O.C. et Taylor R.L., The finite element method, Mc Graw-Hill, 1991, quatrième édition. 